\title{
Measuring the meter: on the constancy of lightness scales seen against different backgrounds
}

\author{
Daniele Zavagno • Olga Daneyko • Tiziano Agostini
}

Published online: 10 November 2010

(C) Psychonomic Society, Inc. 2010

\begin{abstract}
The constancy of a 16-step achromatic Munsell scale was tested with regards to background variations in two experiments. In experiment 1 three groups of observers were asked to find lightness matches for targets in simultaneous lightness displays by using a 16-step achromatic Munsell scale placed on a white, black, or white-black checkered background. In experiment 2 , a yellow-blue checkered background and a green-red checkered background replaced Munsell scales on the black and on the white backgrounds. Significant effects of scale background on matches were found only in experiment 1 , suggesting that background luminance is a crucial factor in the overall appearance of the scale. The lack of significant differences in experiment 2 , however, may stand for an overall robustness of the scale with respect to background luminance changes occurring within certain luminance ranges.
\end{abstract}

Keywords Lightness constancy - Lightness scales ·

Matching method

\section{Introduction}

In everyday life measurement activity is a most pervasive one. People easily and quickly use different instruments to measure the physical property (size, weight, etc.) of

D. Zavagno $(\bowtie)$

Department of Psychology, University of Milano-Bicocca,

Viale dell'Innovazione, 10,

20126, Milano, Italy

e-mail: daniele.zavagno@unimib.it

O. Daneyko · T. Agostini

Department of Psychology, University of Trieste,

Trieste, Italy something in order to find out how things really are. For instance, one can measure the length and the width of a desk with a meterstick, and eventually establish whether the size of the desk matches that of a colleague in another office. People are rather confident about the reliability of their measurements because they trust their tools: they intuitively consider them to be robust with respect to environmental changes. For instance, they are quite sure that differences in office sizes have no effect on the measurements of desks with a meterstick. On the other hand, if someone were simply asked to visually judge whether two desks placed in different rooms were equal in size, room size differences might have an affect on desk size judgments.

Measurement activity is even more pervasive in scientific research. In vision sciences in particular, besides knowing the physical dimensions and/or intensities of stimuli, we most often like to assess the magnitude of their phenomenal outcome. In research on lightness (achromatic surface color), phenomenal outcome is most often measured using matching methods. These methods come essentially in two forms: a subject can either adjust a test stimulus until its lightness (shade of gray) matches a standard stimulus, or a subject can pick a match from an achromatic reflectance scale that matches the lightness of a standard stimulus. The present study addresses the reliability of this second type of measurement, which for sake of brevity we call the matching paradigm.

Achromatic lightness scales

Ever since Burzlaff (1931) first used the matching paradigm, the method has gained in popularity among vision scientists studying lightness constancy and its 
numerous failures. There are basically two reasons behind this popularity:

(1) Matching is a natural operation performed commonly in everyday life (Zwislocki, 1991). When required to "pick a match," participants in an experiment do not find the task unusual or difficult to understand, even when the matching task itself is revealed to be an impossible one (Jandó, Agostini, Galmonte, \& Bruno, 2003).

(2) Reflectance scales are now practically ready-made: one just needs to select appropriate achromatic papers from the Natural Colour System ${ }^{\circledR}$ (NCS) or the Munsell ${ }^{\circledR}$ Color-Order System, cut them into a suitable size and order them from black to white. An appropriate selection of reflectances should guarantee lightness interval scales. Both systems, in fact, were devised to organize color on a perceptual base but in rigorous psychophysical terms (Hård, 1966; Judd, 1940; Judd \& Nickerson, 1975; Munsell, 1916; Nickerson, 1940).

The psychophysics that sustain these color systems do not, however, guarantee what the colors will look like when they are observed outside the psychophysical setup where they were measured. With respect to achromatic color scales, when the matching paradigm is used, matches refer to lightness, not to reflectance. In other words, to the observer the achromatic reflectance scale is a lightness scale. This naked fact implies that the visual outcome of the steps that build up an achromatic reflectance scale can be influenced by environmental factors just like the lightness of any other achromatic surface. In particular, the lightness of a surface is sensitive to two variations in the visual field (Gilchrist, Kossifidis, Agostini, Li, Bonato, Cataliotti, Spehar, Annan, \& Economou, 1999): variations in illumination (which may determine changes in both the luminance of the surface and of its surround) and variations in luminance in the surrounding field (which do not affect the luminance of the surface).

If achromatic color scales are to be used as tools to study lightness perception, we ought to understand their eventual limitations. In other words, we need to find out how robust these scales are with respect to changes in the environment. This work addresses the issue of constancy for achromatic color scales (i.e., lightness scales) seen against different types of backgrounds.

In our experiments we used 16-step lightness scales derived from Munsell Neutral Value papers. While both the aforementioned color systems are equally valid in studying color perception, the Munsell system is tacitly recognized as a standard in lightness studies. The reason is probably related to the fact that Munsell Neutral Value papers come in a greater number of reflectances and can be found in both glossy and matte finish, while NCS papers are only available in glossy finish and with a smaller range of shades of gray. While the second aspect can be undermined - 16-step achromatic Munsell scales are the most used - a glossy finish can determine highlights on stimuli that can interfere with the matching task, whereas the problem is minimized with matte papers.

The unmatchable problem

The popularity of the matching paradigm with Munsell scales among vision scientists researching lightness is such that raw data are sometimes reported in terms of Munsell values. Nevertheless, there are several researchers who do not share the same enthusiasm for the matching paradigm. For instance, Blakeslee, Reetz, and McCourt (2009) recently argued that a 16-step Munsell scale may not be sensitive enough to capture finer differences among the appearance of stimuli. However, experiments conducted with a 35-step Munsell scale show that fine distinctions can be measured, with the advantage of reducing the entity of standard errors with respect to adjustment methods (Zavagno, Annan, \& Caputo, 2004).

We are aware of three works in which the Munsell scale is an object of study. The earliest is by Jacobsen and Gilchrist (1988), who showed that data obtained with a Munsell scale are substantially equivalent to data obtained with a lightness adjustment method. The latest study is by Economou, Zdravkovic, and Gilchrist (2007), who compared lightness matches for a simultaneous lightness contrast display obtained in three different conditions: paper display and paper Munsell scale; computer display and computer simulated Munsell scale; computer display and paper Munsell scale. In all three conditions Munsell scales were seen against white-black checkered backgrounds. No significant differences were reported for matches between conditions.

The study by Jandó, Agostini, Galmonte, and Bruno (2003) dealt instead with the "unmatchable problem," i.e., the impossibility to match a test stimulus that is an increment to its background to a standard stimulus that is a decrement to its background, and vice versa. While the issue is problematic to both the matching paradigm and the adjustment matching methods (Bruno, 1992; Bruno, Bernardis, \& Schirillo, 1997; Whittle \& Challands, 1969), Jandó et al. confronted the problem only with Munsell scales simulated on a CRT. In their study the simulated scales were seen on a black background, white background and a split black-white background. The purpose of the study, however, was not to test the robustness of Munsell scale per se, as seen against different backgrounds, but to determine the possibility of an extended achromatic color scale in order to find a solution to the unmatchable problem. Apart from the differences in scopes, and the fact 
that CRT scales present other problematic issues (for instance target edges are physically enhanced; sometimes unwanted luminance ramps appear when configurations consist of sharp luminance changes) that just add on to the aspects we studied in this work; Jandó et al. claim that "matching scales presented on different backgrounds provide equally reliable measurements of surface color within a certain range" (p. 80).

Measurements, however, are reliable only if the meters used are relatable. For instance, one can measure a desk with a ruler or with a meterstick, and thus have an output in inches or centimeters. We can easily convert such units into one another because they are linear units placed on a ratio scale: rulers and metersticks can be used interchangeably because one can measure the other, and there is no spatial compression or expansion. Are lightness scales, the 'meter' commonly used to study lightness perception, robust to variations in the background luminance against which they are seen? In other words, is there any spatial compression or expansion among the steps that build the scale when this is placed on different backgrounds? It might be that the same Munsell scales placed on different luminance backgrounds appear different, and such differences may reflect a discrepancy between the psychophysical distances among the steps of the scales.

\section{Experiment 1}

In Experiment 1 we tested the robustness of 16-step Munsell scales placed on a white, a black or a whiteblack checkered background. White backgrounds and white-black checkered backgrounds are commonly used in matching experiments, whereas black backgrounds are not so common (Jandó et al., 2003; Agostini \& Galmonte, 2002). Nevertheless, there is no deeply rooted theoretical reason why the latter should not be equally employed. However, Economou et al. (2007) noted that if a Munsell scale were placed on a white background, one "would expect the target on the white background to be correctly matched" [in simultaneous lightness contrast displays] (p. 4). The same logic can be applied with reference to a target on a black background when the Munsell scale is placed on a uniform black background.

\section{Methods}

\section{Participants}

Participants were 48 undergraduate students (39 female) from the University of Milano-Bicocca who volunteered to take part in the experiment. Participants were randomly assigned to one of three groups. Each group used a Munsell scale on a different background.

\section{Stimuli}

Three Munsell scales were prepared by cutting rectangular patches $(6 \times 2.2 \mathrm{~cm})$ from 16 Neutral Value Munsell papers ranging from 2.0 to 9.5 . Each set of 16 Munsell Neutral Value steps was placed in a row (distance of $0.3 \mathrm{~cm}$ between each pair of patches) and centered on top of a background that measured $11.3 \times 41.3 \mathrm{~cm}$. One background was white $\left(2,110 \mathrm{~cd} / \mathrm{m}^{2}\right)$, one was black $\left(70.3 \mathrm{~cd} / \mathrm{m}^{2}\right)$, and one was made of white and black checks $(2,110$ and $70.3 \mathrm{~cd} / \mathrm{m}^{2}$, respectively) that measured $1.56 \times 1.56 \mathrm{~cm}$. Munsell values were printed with the Arial 24-point font on a white background $(1.14 \times 38.8 \mathrm{~cm})$ positioned $0.85 \mathrm{~cm}$ below the Munsell steps. Steps were organized from blackleft $\left(76 \mathrm{~cd} / \mathrm{m}^{2}\right)$ to white-right $\left(1,870 \mathrm{~cd} / \mathrm{m}^{2}\right)$. The black and white-black checkered backgrounds were printed on onesided Epson heavy-weight matte paper.

Classic simultaneous lightness contrast (SLC) displays were used as comparison stimuli (Fig. 1), and consisted of two targets $(2.2 \mathrm{~cm} \times 2.2 \mathrm{~cm})$ cut from Munsell papers, placed with one on a white background $\left(20 \times 20 \mathrm{~cm}, 2,400 \mathrm{~cd} / \mathrm{m}^{2}\right)$ and the other on a black background $\left(68.7 \mathrm{~cd} / \mathrm{m}^{2}\right)$. A target could have a Munsell value of $6.0\left(\mathrm{R} 30 \%, \mathrm{~L} 900 \mathrm{~cd} / \mathrm{m}^{2}\right)$ or $6.5(\mathrm{R} 36.2 \%, \mathrm{~L}$ $\left.1,090 \mathrm{~cd} / \mathrm{m}^{2}\right)$. There were two possible target configurations: "same" (when targets of the same reflectance were presented simultaneously) and "different" (when targets of different reflectance were presented simultaneously); thus, in total there were four SLC displays. The purpose of using SLC displays with different targets is that psychology students are usually familiar with the SLC illusion, and not infrequently they are prone to the stimulus error, which consists in referring what they know about the stimulus (i.e., that two targets are physically equal) rather than what they actually see (Kanizsa, 1979). By employing configurations in which targets can actually be different in luminance, we hope to avoid this type of problem, noticed also by other researchers (Cataliotti \& Becklen, 2007). We chose two reflectance levels that are relatively close but noticeably different to enhance the effect of this, which may be considered a nuisance variable (McBurney \& White, 2007).

Displays and scales were placed on a self-adhesive corklike poster board mounted on a perpendicular wooden panel. Outlined corners were drawn on the board to secure correct placement of SLC displays and scales throughout the experiment. The scale was placed $12 \mathrm{~cm}$ below the SLC display, and both were illuminated by the same spotlight (Spotlight Mini Profile ME with $20^{\circ}$ objective, $250 \mathrm{~W}$, color temperature $5900 \mathrm{~K}$ ) placed on a telescopic stand at $2.2 \mathrm{~m}$ from the ground and $1.5 \mathrm{~m}$ behind the observer. The geometry of the projected light explains the luminance differences between scales and SLC displays: the steps 6.0 


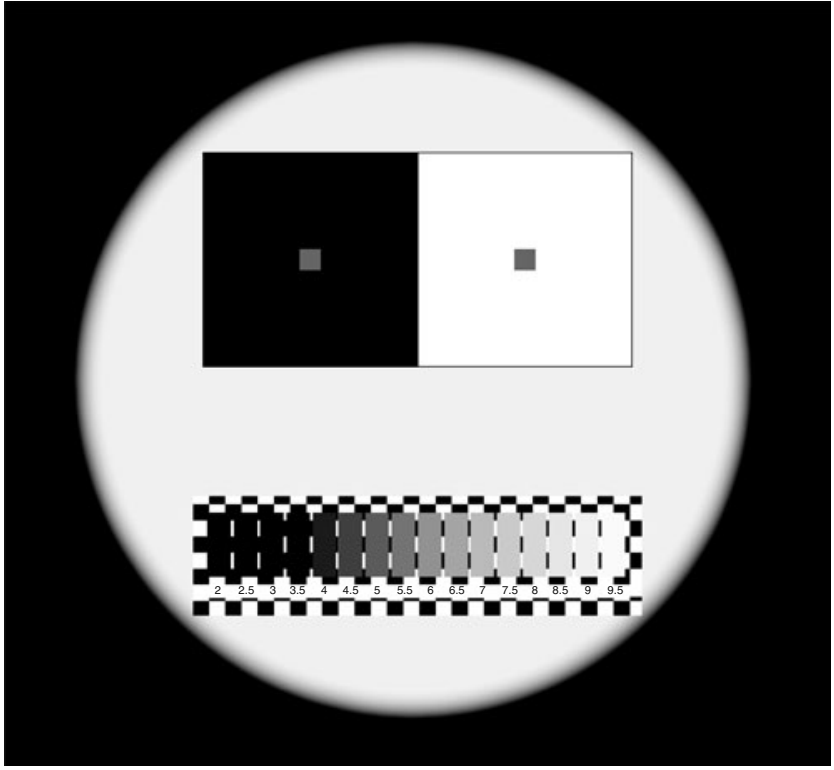

Fig. 1 Example of the experimental setup

and 6.5 on the Munsell scales had luminance respectively of 791 and $980 \mathrm{~cd} / \mathrm{m}^{2}$. However, the luminance differences between SLC targets and corresponding Munsell scale steps are negligible factors in this experiment because they were kept constant in all conditions.

\section{Procedure}

The experiment was conducted in a dark laboratory illuminated only by the same spotlight that illuminated the stimuli. Participants viewed stimuli at a distance of $114 \mathrm{~cm}$. Viewing distance was secured by a chinrest adjustable in height. Participants were informed that their task consisted of finding the match for each square target they saw in the SLC displays. If they could not find a perfect match they were instructed to find the closest match possible. SLC displays were presented in random order twice. During the second presentation, displays were positioned upside down with respect to the previous presentation (also SLC background positions were randomized across the two presentations).

\section{Results}

Results of experiment 1 are shown in Fig. 2. A $2 \times 2 \times 2$ ANOVA for repeated measures (SLC Background $\times$ Target $\times$ Target Combination), with Munsell scale Background as between factor, was conducted on the data: all within factors produced significant main effects $(\mathrm{p}<0.0001)$, as expected, except for Target Combination (same-different) $\left(\mathrm{F}_{1,45}=\right.$ $0.55, p=0.4$ ). Between-factor Munsell scale Background also determined a significant effect on lightness matches $\left(\mathrm{F}_{2}\right.$, $\left.{ }_{45}=7.29, p=0.0018\right)$.
Since the factor Target Combination did not determine significant effects, we focused our attention on the results for "targets same," i.e., the two configurations in which Ts were equal in luminance. With respect to T-6.0 on the black background of the SLC display, unpaired t-tests show that matches obtained with the Munsell scale on the white background were significantly different from both matches obtained with Munsell scales on the white-black checkered and on the black backgrounds (respectively: $\mathrm{t}_{30}=2.67, p=$ $0.013 ; \mathrm{t}_{30}=3.7, p=0.0008$ ), while matches obtained with Munsell scales on the white-black checkered and on the black backgrounds are statistically indistinguishable $\left(\mathrm{t}_{30}=\right.$ $1.11, p=0.27$ ). As for T-6.0 on the white background, there is a significant difference only between matches carried out with Munsell scales on the white and on the black backgrounds $\left(\mathrm{T}_{30}=2.31, p=0.02\right)$. Unpaired t-tests for $\mathrm{T}$ 6.5 on the black background show that there is a tendency towards a significant difference only between matches obtained with Munsell scales on the white and on the black backgrounds $\left(\mathrm{t}_{30}=1.95, p=0.06\right)$. Unpaired $\mathrm{t}$-tests for T-6.5 on the white background show statistical differences between matches obtained with Munsell scales on the white and on the white-black checkered backgrounds $\left(\mathrm{t}_{30}=2.48, p=\right.$ 0.018 ), and between matches with Munsell scales on the white and the black backgrounds $\mathrm{t}_{30}=2.04, p=0.05$ ).

\section{Discussion}

Results suggest that the appearance of the Munsell scale is affected by the background against which it is seen. However, it is important to notice that scale backgrounds did not affect the magnitude of the contrast effects much: in terms of perceived reflectance, the differences between contrast effects across scales were $\pm 5.6 \%$ for targets 6.0 and $\pm 3.1 \%$ for targets 6.5 . Instead, scale backgrounds affected the way targets were matched: the scale on the black background induced matches for the target on the black background that were relatively closer to the actual reflectance value on the Munsell scale; contrarily the scale on the white background induced matches for the target on the white background that were relatively closer to the actual reflectance value on the Munsell scale. These findings do not confirm the expectations described by Economou et al. (2007), i.e., that matches for an SLC target would be "correct" when the matching scale is placed on a similar background. We found instead always some deviation from the scale value of the target with a background corresponding to that of the scale. Our results for matches with white-black checkered backgrounds are also intrinsically different from the results reported by Economou et al.: while they report greater deviations for the target on the black background, we found that deviations from the actual scale value were roughly the 
Fig. 2 Mean matches for experiment 1 . The left side of each graph shows results for targets same, the right side for targets different (see text). Dashed lines indicate the actual reflectance value of the targets
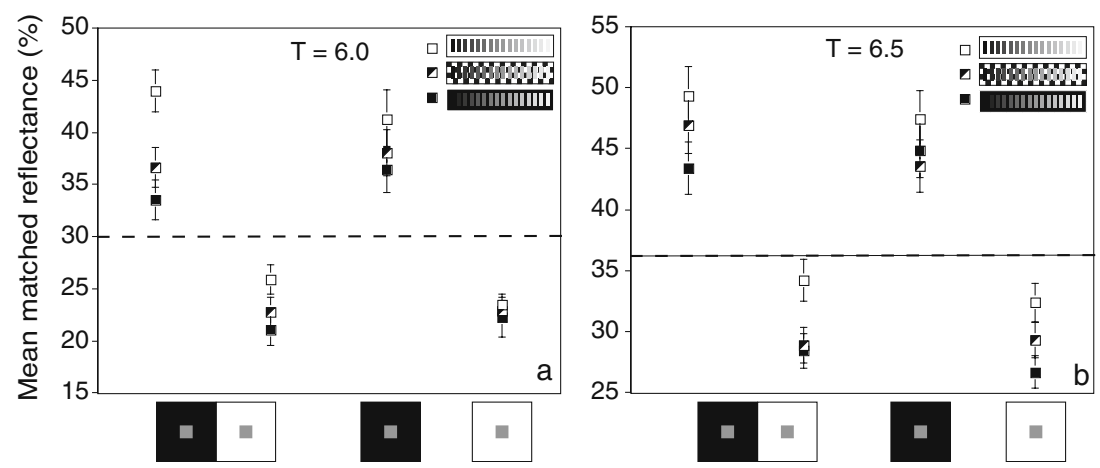

same for the two targets. The differences between our findings and theirs will be discussed in the Conclusions.

Our experiment suggests an important question: do the matching differences we found using different scale backgrounds reflect a specific matching strategy (for example, it is easier to find a match for the target if its background is similar to that of the scale), or do they reflect the fact that the scales appear different?

The first alternative is intuitively reasonable. However, if it were to be confirmed, according to our data it would mean that observers first judge the difference in lightness between two targets and then based on the scale they use they select the lightness steps that better express such difference between the two targets. This second step is "biased" by the background of the scale. Hence matches would not be based on the specific appearance of a target, but on how different or similar targets appear with respect to each other (i.e., the magnitude of the contrast illusion for a given SLC display). This hypothesis does not assume that a same scale appears different on different backgrounds, but that matching is easier for a target seen against a background similar to the one behind the scale.

The second alternative appears to be more grounded in the literature. According to some studies, incremental targets (targets with higher luminance than the background) elicit luminance matches (i.e., brightness matches), whereas decremental targets elicit ratio matches (i.e., lightness matches) (Jacobsen \& Gilchrist, 1988; Whittle \& Challands, 1969). This empirical fact, however, holds only for very simple configurations in which lightness and brightness are collapsed in the same feature (Whittle, 2004), for example, in displays with low articulation or with poor illumination information. Apart from the fact that the participants in our experiment were explicitly told to find on a Munsell scale the "color" of a target - "each target is cut from gray papers, a sample of which are part of the matching scale; find the match of the target by finding the corresponding gray color on the scale" - our Munsell scales are highly articulated structures.

Nevertheless, a plausible hypothesis is that the overall organization of the scale in combination with the luminance of the background delivers brightness information to the visual system, and that this information affects the way scales are seen. With brightness information we do not simply refer to "perceived luminance," but also to information about perceived intensities, that is information relative to our visual experiences of luminosity (Zavagno \& Caputo, 2005) and illumination (Whittle, 2004; Zavagno \& Daneyko, 2008b). Hence, incremental and decremental Munsell scales would determine different matches for the same stimulus configurations because of illumination information that affects the way the scales appear. Such information would presumably consist of the overall organization of the scale (from dark to light), of the individual ratios between the steps of the scales and the background, and of the ratio between the background and the scale as a Gestalt (Fig. 3). This hypothesis is supported by data from experiments in which stimuli consisted of socalled "luminance pseudo-ramps" (pseudo-ramps are made of discrete luminance steps that are not adjacent to each other, organized in a row from the darkest to the brightest step; Zavagno \& Daneyko, 2008a), which determine brightness effects similar to those found with actual gradients (Agostini \& Galmonte, 2002; Daneyko \& Zavagno, 2008a).

This second hypothesis is consistent with the results obtained with the Munsell scale placed on the white-black checkered background: matches fall somewhat in between those obtained with the other two scale backgrounds. It could be that the highly contrasted articulation of the background reduces the saliency of the illumination information, or it could be that the luminance of the white-black checkered background is averaged by the visual system to determine some level of illumination, as proposed by Helmholtz, Hering, Katz, Mach (Gilchrist, 2006) and Musatti (1953). However, more recent studies do not support the averaging hypothesis: it was found that backgrounds differently articulated in luminance are not equivalent to homogeneous backgrounds in which luminance is the average of the first (Brown \& MacLeod, 1991; Bruno, 1992), nor are luminance-articulated backgrounds with the same average luminance but different spatial 
Fig. 3 A lightness scale placed on a uniform black or white background determines luminance pseudo-ramps (see text), the appearance of which is presumably affected by a system of ratios with the background

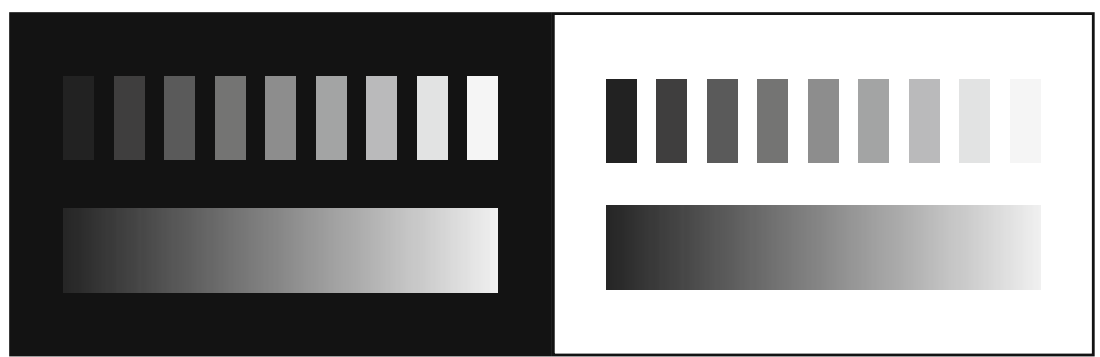

frequency equivalent for matching purposes (Schirillo \& Shevell, 1996).

\section{Experiment 2}

In Experiment 2 we tested the effect of chromatic checkered backgrounds on lightness matches. The aim of this experiment was not to find chromatic effects on the Munsell scale caused by backgrounds made of different hues, but to verify if the chromatic and luminance components of such backgrounds had an effect on lightness matches.

\section{Methods}

\section{Participants}

Participants were 30 undergraduate students (27 female) from the University of Milano-Bicocca who did not take part in the previous experiment. Participants were randomly assigned to one of three groups; each group used a Munsell scale on a different background.

\section{Stimuli}

The experimental setup and procedure were similar to those in experiment 1, except for the level of illumination (a change in laboratory determined different luminance intensities, although the equipment employed was the same) and the backgrounds of the Munsell scale.

Munsell scales were seen against three checkered backgrounds: blue-yellow, green-red and white-black. Except for background hues and the luminance range of the scales (Munsell $2.0=19.3 \mathrm{~cd} / \mathrm{m}^{2}$; Munsell $9.5=$ $550 \mathrm{~cd} / \mathrm{m}^{2}$ ), Munsell scales were equal in size and organization to those employed in Experiment 1. The luminance values of the blue and yellow checks were respectively 38.5 and $360 \mathrm{~cd} / \mathrm{m}^{2}$, those of green and red checks were 143 and $100 \mathrm{~cd} / \mathrm{m}^{2}$, and those of the white and black checks were 570 and $17.8 \mathrm{~cd} / \mathrm{m}^{2}$.

The luminance values for T-6.0 and T-6.5 were respectively 250 and $310 \mathrm{~cd} / \mathrm{m}^{2}$; the corresponding M-6.0 and M-
6.5 on the Munsell scales had luminance values 185 and $224 \mathrm{~cd} / \mathrm{m}^{2}$. The luminance values of the white and the black SLC backgrounds were respectively 695 and $21.7 \mathrm{~cd} / \mathrm{m}^{2}$.

Results

The results of experiment 2 are plotted in Fig. 4 . A $2 \times 2 \times$ 2 (Target $\times$ Target Combination $\times$ Target Background) ANOVA for repeated measures, with Scale Background as between factor, was conducted on the data: as expected, main effects were found for the factor Target and Target Background $(p<0.0001)$, but not for Target Combination $\left(\mathrm{F}_{1,27}=0.53, p=0.4\right)$. The between-factor Scale Background did not produce significant effects on lightness matches $\left(\mathrm{F}_{2,27}=0.13, p=0.8\right)$. None of the interactions were significant $(p>0.18)$. Unpaired t-tests were conducted on the data, but no significant differences were found.

\section{Discussion}

At a first glance, the results seem to support the average background luminance hypothesis that was proposed by several authors between the nineteenth and twentieth centuries to explain how the visual system derives information about field illumination. Yet if we compare the Michelson contrast of the three checkered backgrounds, only the blue-yellow and black-white are relatively similar: respectively 0.8 and 0.9 versus the 0.17 of the red-green background. If each background were converted into its equivalent achromatic mean luminance, the blue-yellow would roughly correspond to a background of $200 \mathrm{~cd} / \mathrm{m}^{2}$ and the black-white to a background of $217 \mathrm{~cd} / \mathrm{m}^{2}$, whereas the red-green would roughly correspond to a background of only $121 \mathrm{~cd} / \mathrm{m}^{2}$. A difference of about $80 \mathrm{~cd} / \mathrm{m}^{2}$ is not so negligible; hence, it seems that the Munsell scale is rather robust to both background hue and average luminance differences.

If the background average luminance hypothesis holds, it would mean that the visual system is rather tolerant to background average luminance variations occurring within a certain range, at least with respect to the appearance of a highly articulated group of surfaces that form a Gestalt unit, such as the Munsell scale. In other words, a Munsell scale 
Fig. 4 Mean matches for experiment 2. The left side of each graph shows results for targets same, the right side for targets different (see text). Dashed lines indicate the actual reflectance value of the targets
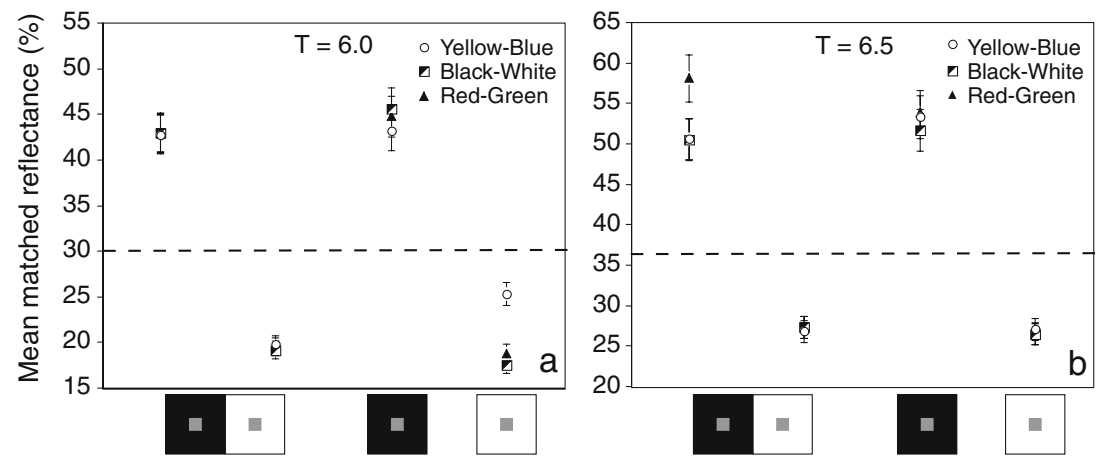

shows higher constancy because of its articulation. This hypothesis is supported by empirical studies in which highly articulated patterns show a higher degree of lightness constancy (Gilchrist \& Annan, 2002; Gilchrist et al., 1999).

A similar tolerance to field changes can be advanced also with respect to illumination variations on the Munsell scale: an ANOVA for repeated measures conducted on the data from experiments 1 and 2 for white-black checkered backgrounds, with Experiment-Illumination as between factor, yielded no significant effect $\left(\mathrm{F}_{1,27}=0.805, p=0.3786\right)$.

Nevertheless, the graphs displayed in Fig. 5 show a tendency for Ts on the black background to become lighter and for Ts on the white background to become darker when the illumination of both the background and the target are reduced. This finding is rather curious and should be addressed separately with a new series of experiments aimed to study the effect of illumination differences on both the Munsell scale and SLC displays.

\section{Conclusions}

We tested the general robustness of a 16-step achromatic Munsell scale with regards to background luminance and hue variations. Depending on how one wants to consider the results, two different conclusions can be drawn.

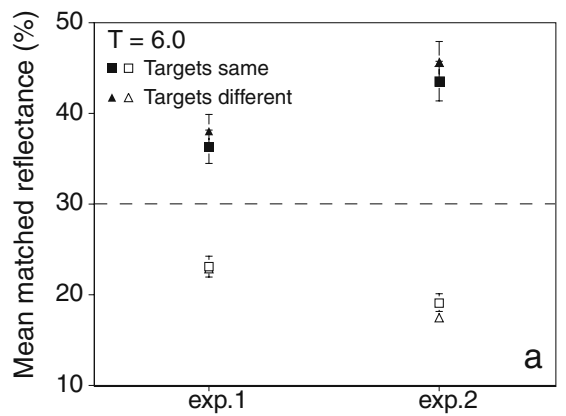

Fig. 5 Comparisons between matches in experiments 1 and 2 for targets 6.0 (a) and 6.5 (b) with black and white checkered backgrounds. Black symbols and white symbols indicate respectively targets on a black and
On one hand, according to the results of experiment 1, the Munsell scale is not a reliable tool, in the sense that its appearance is influenced by the luminance of its background. A good measurement tool should instead show a high degree of constancy despite significant environmental variations. This does not mean that the matching paradigm is not useful or that it returns "bad" data. On the contrary, we believe that the paradigm is very useful and that, given its simplicity, it is a powerful tool in lightness studies. Yet researchers ought to be aware of its limitations: the matching paradigm returns data that can be used to measure the relative magnitude of lightness illusions, but not the absolute magnitudes of such illusions. For instance, Economou et al. (2007) claim that the error (the deviation from the actual reflectance of a target) in simultaneous lightness contrast displays is greater for the target on the black background. We found this to be true when the Munsell scale is seen against a white background, but we also found the opposite when the same scale is seen against a black background. When the scale is seen on a white-black checkered background, we found the magnitudes of deviations to be approximately equal for the two targets. Economou et al. (2007), however, also employed Munsell scales with white-black checkered backgrounds. The difference in findings cannot be attributed to the fact that we used Munsell papers 6.0 and 6.5 for our SLC targets, while they employed Munsell values 5.0 (experiment 1, conducted both on paper and on a CRT), and also simulated

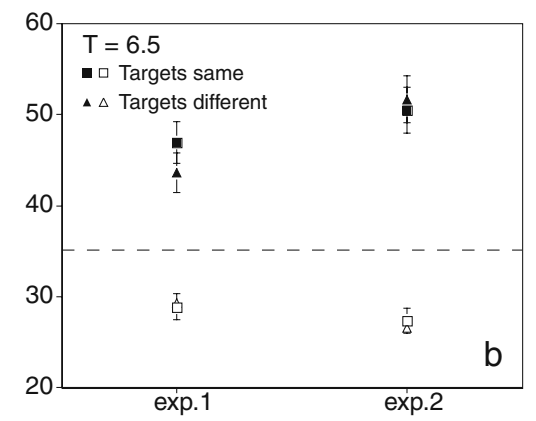

targets on a white background. Squares indicate targets same; triangles indicate targets different. The dashed line indicates the actual reflectance value of the targets 
Munsell values 3.0, 7.0 and 8.0 (experiment 2 on a CRT). Our data for Munsell targets 6.5 should somewhat resemble their data for Munsell targets 7.0. However, the two sets of data are quite different. It should be noticed that Economou et al. conducted two runs for each pair of targets in their experiment 2 , with slightly different luminance values for the targets in the two runs (in one run the simulated Munsell value $7.0 \mathrm{had}$ a luminance of $12 \mathrm{~cd} / \mathrm{m}^{2}$, in the other run it had luminance $5.48 \mathrm{~cd} / \mathrm{m}^{2}$ ), which determined two different patterns of results: one in which targets' deviations from the scale value are similar (run 1), and the other in which the deviation of the target on the white background is greatly reduced (run 2). An interesting fact is that the magnitudes of the contrast effects in the two runs, plotted in log reflectance values, are roughly the same. While the authors make no mention about whether the mean matches were statistically indistinguishable or different across runs, we assume that also the simulated Munsell scale was somewhat different from run 1 to run 2 in terms of luminance values.

We believe that the differences between our data and the data published by Economou et al. (2007) depend upon factors other than the Munsell value used for SLC targets. Such factors could be the level of perceived illumination (the CRT display is a special "environment"), whether the scale is seen within the same framework of illumination, etc. Hence, comparisons between experiments can be tricky and lead to inappropriate generalizations (Daneyko \& Zavagno, 2008b). It would be wise instead to model data with a certain degree of caution: Munsell scales do not return "absolute" lightness data, but relative lightness data, because the scale itself is a phenomenon that appears to be sensitive to luminance variations in the background, and probably to other factors not contemplated in this study. We believe that the same caution should be used towards data obtained with adjustment matching methods for the same reason: the appearance of the adjustable patch is influenced by its background, even when the background is a checkerboard (Schirillo \& Shevell, 1996).

On the other hand, the results from experiment 2 suggest also that the Munsell scale is rather robust when seen against articulated backgrounds. This, however, does not necessarily mean that a Munsell scale on a checkered background shows its "veridical" face. In other words, we are not sure that each step of the scale is the absolute transformation of the reflectance value into the appropriate perceived reflectance. First of all, more experiments need to be carried out to understand why differences were not found in Experiment 2. Does the visual system average the lightness of articulated backgrounds? If so, is averaging "simple" or more complex, taking into account also spatial frequency (Schirillo \& Shevell, 1996), a factor that we did not manipulate in these experiments? Or is it that the articulation of the back- ground is the key to the scale's constancy, regardless of the background's average luminance?

Secondly, we still do not know what the effects of illumination on the scales are (see Fig. 5) or how differences in illumination might affect matching results with scales on different backgrounds. We are currently conducting experiments to test the robustness of the achromatic Munsell scale with respect to variations in illumination.

\section{References}

Agostini, T., \& Galmonte, A. (2002). A new effect of luminance gradient on achromatic simultaneous contrast. Psychonomic Bulletin \& Review, 9, 264-269.

Blakeslee, B., Reetz, D., \& McCourt, M.E. (2009). Spatial filtering versus anchoring accounts of brightness/lightness perception in staircase and simultaneous brightness/lightness contrast stimuli. Journal of Vision, 9(3):22, 1-17, http://journalofvision.org/9/3/ 22/, doi:10.1167/9.3.22

Brown, R.O., \& MacLeod, D. I. A. (1991). Induction and constancy for color saturation and achromatic contrast variance. Investigative Ophthalmology and Visual Science, 32 Supplement, 1214.

Bruno, N. (1992). Lightness, equivalent backgrounds and the spatial integration of luminance. Perception, 21, ECVP Abstract Supplement, 80 .

Bruno, N., Bernardis, P., \& Schirillo, J. (1997). Lightness, equivalent backgrounds, and anchoring. Perception \& Psychophysics, 59, 634-654.

Burzlaff, W. (1931). Methodologische Beiträge zum Problem der Farbenkonstanz" [Methodological notes on the problem of color constancy]. Zeitschrift für Psychologie, 119, 117-235.

Cataliotti, J., \& Becklen, R. (2007). Single dissociations between lightness contrast effects. Perception, 36, ECVP Abstract Supplement, 79 .

Daneyko, O., \& Zavagno, D. (2008a). Simultaneous lightness contrast with non-adjacent luminance ramps and Gelb illumination. Perception, 37 ECVP Abstract Supplement, 104.

Daneyko, O., \& Zavagno, D. (2008b). The concept of error in visual perception: The case of simultaneous lightness contrast. Teorie \& Modelli, n.s. 13, 175-184.

Economou, E., Zdravkovic, S., \& Gilchrist, A. (2007). Anchoring versus spatial filtering accounts of simultaneous lightness contrast. Journal of Vision, 7(12):2, 1-15, http://journalofvision. org/7/12/2/, doi:10.1167/7.12.2.

Gilchrist, A. (2006). Seeing black and white. New York: Oxford University Press.

Gilchrist, A., \& Annan, V. (2002). Articulation effects in lightness: Historical background and theoretical implications. Perception, 31, 141-150.

Gilchrist, A., Kossifidis, C., Agostini, T., Li, X., Bonato, F., Cataliotti, J., et al. (1999). An anchoring theory of lightness perception. Psychological review, 106, 795-834.

Hård, A. (1966). Philosophy of the Hering-Johansson natural colour system. Farbe, 15, 287-295.

Jacobsen, A., \& Gilchrist, A. (1988). The ratio principle holds over a million-to-one range of illumination. Perception \& Psychophysics, 43, 1-6.

Jandó, G., Agostini, T., Galmonte, A., \& Bruno, N. (2003). Measuring surface achromatic color: Toward a common measure for increments and decrements. Behaviour Research Methods, Instruments, \& Computers, 35, 70-81. 
Judd, D. B. (1940). The Munsell colour system. Journal of the Optical Society of America, 30, 574.

Judd, D. B., \& Nickerson, D. (1975). Relation between Munsell and Swedish natural colour system scales. Journal of the Optical Society of America, 65, 85-90.

Kanizsa, G. (1979). Organization in vision. Essays on Gestalt Perception. New York: Praeger.

McBurney, D. H., \& White, T. L. (2007). Research methods. Belmont: Thomson Wadsworth.

Munsell, A. H. (1916). A colour notation. Boston: Geo. H Ellis Co.

Musatti, C. L. (1953). Luce e colore nei fenomeni del "contrasto simultaneo", della "costanza" e dell'"eguagliamento" [Light and color in phenomena of "simultaneous contrast", "constancy", and "equalization"]. Archivio di Psicologia, Neurologia e Psichiatria, 14, 544-577.

Nickerson, D. (1940). History of the Munsell colour system and its scientific application. Journal of the Optical Society of America, $30,575-586$.

Schirillo, J., \& Shevell, S. (1996). Brightness contrast from inhomogeneous surrounds. Vision Research, 36, 1783-1796.
Whittle, P. (2004). Contrast brightness and ordinary seeing. In A. Gilchrist (Ed.), Lightness, brightness, and transparency (pp. 111158). Hillsdale: Erlbaum.

Whittle, P., \& Challands, P. D. C. (1969). The effect of background luminance on the brightness of flashes. Vision Research, 9, 10951110 .

Zavagno, D., Annan, V., \& Caputo, G. (2004). The problem of being white: Testing the highest luminance rule. Vision, 16, 149-159.

Zavagno, D., \& Caputo, G. (2005). Glowing greys and surface white: The photogeometric factors of luminosity perception. Perception, 34, 261-274.

Zavagno, D., \& Daneyko, O. (2008a). The effect of luminance pseudo-ramps on simultaneous lightness contrast. Perception, 37 ECVP Abstract Supplement, 147.

Zavagno, D., \& Daneyko, O. (2008b). When figure-ground segmentation modulates brightness: The case of phantom illumination. Acta Psychologica, 129, 166-174.

Zwislocki, J. J. (1991). Natural measurement. In S. J. Bolanowski \& G. A. Gescheider (Eds.), Ratio scaling of psychological magnitude (pp. 18-26). Hillsdale: Lawrence Erlbaum Associates. 